\title{
The Psychological Changes of Horticultural Therapy Intervention for Elderly Women of Earthquake-Related Areas
}

\section{Yuka Kotozaki}

Smart Ageing International Research Center, Institute of Development, Aging, and Cancer, Tohoku University, Sendai, Japan

\begin{abstract}
Despite the passage of time, some people who experienced the Great East Japan Earthquake are still living with the emotional trauma and stress. We provided horticultural therapy as an intervention to 39 elderly women with earthquake stress living in the affected areas of the coastal areas of the Great East Japan Earthquake. The participants were divided into two groups, an intervention group $(n=20)$ and a control group $(n=19)$. The intervention group underwent eight weeks of horticultural intervention. On the other hands, the control group underwent eight weeks of stress control education. After two months of horticultural therapy intervention, the Clinician-Administered PTSD Scale (CAPS) total score, Geriatric Depression Scale (GDS) score, the Posttraumatic Growth Inventory (PTGI-J) score, and the WHO Quality of Life 26 (WHO-QOL26) score in the intervention group improved significantly, and salivary cortisol level in the intervention group also improved significantly. After follow up, CAPS score, GDS score, PTGI-J score, and WHO-QOL26 score (psychological QOL score, social QOL score, environmental QOL score, and global QOL score), and salivary cortisol level in the intervention group was improved or almost the same as the post-intervention scores in the intervention group. These findings suggest that horticultural therapy has an effect on the symptoms of earthquake stress in elderly women, and that this effect may sustain.
\end{abstract}

Keywords: Earthquake; Earthquake-related stress; Horticultural therapy; Intervention; Elderly women

\section{Introduction}

The Great East Japan Earthquake that occurred on March 11, 2011 was the earthquake and tsunami of the largest in the earthquake that occurred in Japan. Despite the passage of time, some people living of the Pacific coast of Tohoku have complained about the mental and physical condition such as insomnia and anxiety. This means that some people who experienced the Great East Japan Earthquake are still living with the emotional trauma and stress. Previous studies report that prevalence of Posttraumatic Stress Disorder (PTSD) ranging from approximately 5 percent to 60 percent is seen in the first 1-2 years after a disaster $[1,2]$. It suggest that mental health problems of survivors are most evident a certain amount of time after a disasters [3]. Additionally, previous studies reported about mental health problems of survivors after a disaster such as a temporary increase in cortisol level [4-7]. The recovery is progressing little by little in the disaster area of Tohoku and it may be said that it is important to medium- to longterm psychological care for the people who live in the disaster area. In this study, as a method of medium- to long-term psychological care for them, we focused on the horticultural therapy.

Horticultural Therapy (HT) is a psychological care method for PostTraumatic Stress Disorder (PTSD) that was developed in the United States for the psychological care and social rehabilitation of disabled soldiers and war veterans with PTSD symptoms after World War II [8]. HT interventions are led by professionals trained to incorporate the use of plants and horticultural education into rehabilitation therapies [8]. The therapy in a group setting improves the participant's communication skills through collaborative horticultural activities [8]. It has been reported that participants begin to identify with plant growth, regain health and motivation. Through such experiences and their association with nature, participants are thought to experience improvemen [9]. It has mainly been developed for elderly adults and people with disabilities $[10,11]$.

Previous studies suggested that HT and exposure to nature can have cognitive [12,13], psychological [13-17], social [18,19], and physical [20] benefits. It also suggested that HT has a positive effect on physiological factors, such as heart rate and salivary cortisol levels [20]. Previous HT studies have utilized psychological measures and observational data. Recently, the study on the effects of HT for earthquake stress reported by our group [21]. However, the person targeted for the study was an adult woman and was not able to examine the elderly woman. Previous study suggest that women are easy to feel anxiety of post-disaster than men [21] and Women are more likely than men to have PTSD after natural disasters [22-25], and low social support is associated with a higher likelihood of PTSD [26,27]. Other studies reported that the elderly people were more likely to develop PTSD and general psychiatric morbidity compared with the young people $[25,28-30]$. We performed an experimental study aimed at elderly woman from 60 to 75 years old because there was no study of effect verification of horticultural therapy for elderly woman who live in disaster area. We hypothesize that HT may help elderly women with earthquake-related stress improve their mental and physical functioning affected due to the traumatic experience.

The purpose of this study was to verify the reduction in the symptoms of earthquake-related stress in elderly women who live in disaster areas of the Pacific coast through HT intervention using psychological measures and salivary cortisol level. Additionally, we investigated the effect of HT on the symptoms of earthquake-related

*Corresponding author: Yuka Kotozaki, Smart Ageing International Research Center, Institute of Development, Aging and Cancer, Tohoku University, 4-1 Seiryomachi, Aoba-ku, Sendai 980-8575, Japan, Tel: +81 (0) 22717 7988; E-mail: kotoyuka@idac.tohoku.ac.jp

Received November 30, 2013; Accepted December 23, 2013; Published December 26, 2013

Citation: Kotozaki Y (2013) The Psychological Changes of Horticultural Therapy Intervention for Elderly Women of Earthquake-Related Areas. J Trauma Treat 3 : 184. doi:10.4172/2167-1222.1000184

Copyright: (c) 2013 Kotozaki Y. This is an open-access article distributed unde the terms of the Creative Commons Attribution License, which permits unrestricted use, distribution, and reproduction in any medium, provided the original author and source are credited. 
stress and the maintenance of its effect after a two-month Follow-Up (FU) period using psychological measures and salivary cortisol level.

\section{Methods}

\section{Participants}

Theparticipantswere womenaged 60-75years old who were residents of the coastal areas of Miyagi Prefecture and had experienced the Great East Japan Earthquake of March 11, 2011. They were recruited through newspaper advertisements distributed in the earthquake-affected areas, to which 100 residents of the coastal areas (from Kesennuma City to Watari town) responded. These 100 applicants were screened for PTSD using a combination of the Mini-International Neuropsychiatric Interview (M.I.N.I.) [31,32] and the Clinician-Administered PTSD Scale (CAPS) [33-35]. In the CAPS, the F1/2 method was used for evaluation, with applicants regarded as symptomatic if they scored $\geq 1$ on frequency and $\geq 2$ on intensity. After the exclusion of 61 applicants who had no PTSD symptoms and a CAPS score of $\geq 40,39$ healthy, right-handed elderly women participated in this study as part of our ongoing project to investigate the associations between brain structure and mental health. All participants who took part in this study also participated in our interventional studies and underwent psychological measures and MRI scans that are not described in this study but were performed together with those described in this study. All participants were diagnosed with a symptom of PTSD on the M.I.N.I., and they had one to two symptoms of all three PTSD symptom clusters, including re-experiencing the event, avoidance, and hyperarousal. The CAPS and M.I.N.I. were administered before and after the intervention and FU. This study was approved by the Research Ethics Committee of Tohoku University Graduate School of Medicine after an ethical screening. Informed consent in writing was obtained from the experimental participants before the start of the experiment. The intervention period was from October 2012 to May 2013.

The study was a randomized, open -label assessors are blinded, crossover trial (RCT), and it was registered in the University Hospital Medical Information Network Clinical Trials Registry (UMIN 000008936). Testers are blind to the study's hypothesis and the group membership of participants. The participants were divided into two groups, an intervention group $(n=20)$ and a control group $(n=19)$, by the permuted block method, and the intervention group underwent eight weeks of horticultural intervention followed by an eight-week FU period. The control group underwent eight weeks of stress control education, followed by eight weeks of horticultural intervention (Figure 1). Although the intervention group had a follow-up period after the intervention period, participants were allowed to keep growing plants during the follow-up period because it could have been stressful or created negative emotions, if we required participants to stop their horticultural activities. In addition, this study design used a design same as our previous study [21].

\section{Description of interventions}

Horticultural intervention (Intervention group): The HT intervention was designed in collaboration with a horticultural therapist and clinical psychologists. This intervention comprised a total of eight weekly sessions ( 60 min each) at a university lab and 15 minutes per day at participants' homes. The sessions at a university lab were comprised of interactive lectures and practical horticultural training. The participants then attended six horticultural lessons, including topics such as designing a garden planter, seeding, watering, weeding, and picking flowers. Participants filled out an HT intervention session checklist after each session as a self-assessment. Participants took care of plants for $15 \mathrm{~min}$ per day at their convenience with horticulture kits provided by the experimenters, and recorded the completion of this task daily on forms provided by the experimenters at the intervention sessions. The participants submitted these forms to the experimenters at the HT intervention session each week.

Stress control education intervention (Control group): The SE intervention session was a 60 -minute session consisting of a lecture regarding stress education, and it was managed by psychological tester studied psychology in college as a whole-time teacher of this intervention. The participants in the control group attended the SE intervention sessions once each week (a total of eight lessons). The video series used in the SE intervention sessions taught participants about the human body, such as stress mechanisms, psychology, and stress management. Participants filled out an SE intervention session checklist after each session. The $2^{\text {nd }}$ session and the $6^{\text {th }}$ session of the HT intervention session and the SE intervention session used the same teaching aid.

Follow-up (Intervention group): At the end of the two-month horticultural intervention, the participants allocated to the intervention group entered a two-month FU period. During this period, they did not receive any specific instructions from the investigators and were asked to lead their normal lives.

\section{PTSD screening: M.I.N.I. and CAPS}

In this study, structured interviews, M.I.N.I. and CAPS, were performed by three psychologists who underwent training before carrying out the actual interviews.

Psychological measures: The following questionnaires were administered three times (pre-intervention, post-intervention, and FU): (a) short version of the Geriatric Depression Scale (GDS) [36,37], (b) the Posttraumatic Growth Inventory (PTGI) [38,39], (c) the World Health Organization Quality of Life 26 instrument questionnaire (WHO-QOL26) [40]. We used the Japanese version of these psychological measures.

\section{Saliva sampling}

We collected saliva samples from participants to measure the salivary cortisol levels. Distressing psychological stimuli are associated with an increased cortisol level [7,41]. In consideration of the participants' circadian cortisol rhythms, we collected all saliva samples

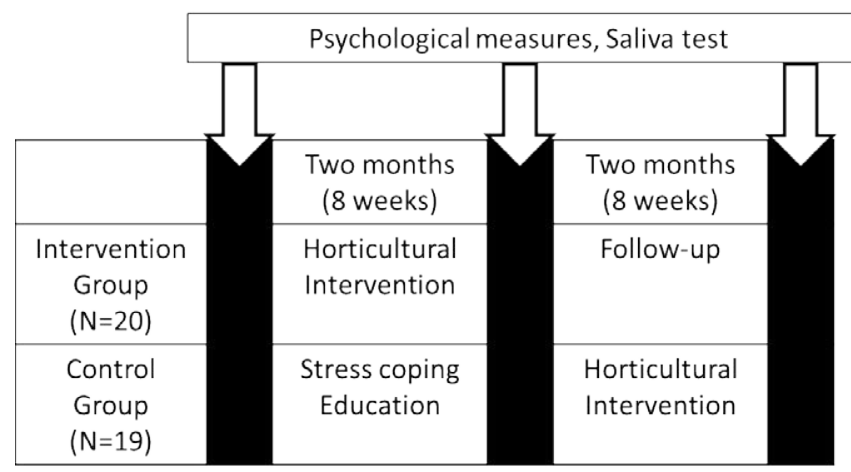

Figure 1: Intervention design. 
at 4:00 p.m. on weekdays both before and after the intervention. We selected 4:00 p.m. because humans are less affected by circadian cortisol rhythms at this time of day [42]. Participants were asked to refrain from drinking, eating, and exercising [43] for two hours before saliva sampling. This method was same method as our previous studies $[7,21]$.

Measure of salivary cortisol: To assess physiological stress, we used the same technique to measure salivary cortisol as described in a previous study $[7,21]$. Saliva samples were collected using the salivette apparatus (Sarstedt, Nümbrecht, Germany). We stored the supernatant solutions in airtight containers at $-80^{\circ} \mathrm{C}$ and measured salivary cortisol using the solutions. We measured salivary cortisol with a semi-microcolumn high-performance liquid chromatography (HPLC) system (Shiseido, Tokyo).

\section{Analytical methods}

The psychological and salivary data were analyzed using the PASW statistical software package (ver. 18 for Windows; SPSS Inc., Chicago, IL, USA). Demographic and clinical data were subjected to the one-way analyses of variance. The one-way analyses of covariance (ANOVA) were conducted with the differences between the pre- and post-intervention scores included as dependent variables and pretest scores as covariates of each psychological measure. Because our primary endpoint of interest was the beneficial effect of intervention training, test-retest changes were compared between the intervention and control groups using one-tailed tests $(p<0.05)$, in the same manner as in previous studies [7,21].

Moreover, changes in the intervention group were confirmed by the analysis of variance of psychological measure scores and salivary cortisol level at three points (pre-intervention, post-intervention, and FU) using repeated-measures ANOVA. A post hoc analysis was carried out by using the Bonferroni's multiple comparison. The significance was established at a level of $\mathrm{p}<0.05$.

\section{Results}

\section{Comparison of an intervention group with a control group (Pre vs. Post)}

Psychological measures: The demographic and clinical data for the study participants are given in Table 1 . The age and ClinicianAdministered PTSD Scale (CAPS) scores did not differ significantly between the intervention group and control group. Comparisons of the psychological changes before and after the intervention between the two groups are shown in Table 2. The intervention group was a significant decrease in the post-intervention CAPS score $[1,37](\mathrm{F}=4.47, p<0.05)$, and the GDS score $(\mathrm{F}[1,37]=5.12, p<0.05)$ compared with the control group. The intervention group also showed a significantly improve in the post-intervention Posttraumatic Growth Inventory (PTGI)-J total scores $(\mathrm{F}[1,37]=6.36, p<0.01)$, WHO-QOL26 Psychological score $(\mathrm{F}[1,37]=5.95 p<0.01)$, WHO-QOL26 Social score $(\mathrm{F}[1,37]=8.96$, $p<0.01)$, WHO-QOL26 Environmental score $(\mathrm{F}[1,37]=4.46, p<0.05)$, and WHO-QOL26 Global score $(\mathrm{F}[1,37]=10.98, p<0.001)$ compared with the control group.

Salivary cortisol level: The results of comparisons of salivary cortisol levels measured pre- and post-intervention are shown in Table 2 . The intervention group was a significant decrease in salivary cortisol $[1,37](\mathrm{F}=4.83, \mathrm{p}<0.05)$, indicating a reduction of stress in this group compared with the control group.

\section{Psychological changes of an intervention group (The effectiveness of HT intervention)}

Psychological measures: Changes in the various psychological measures of the intervention group are shown in Table 3. CAPS scores showed that the main effect of time (pre-intervention, postintervention, and FU) was significant $[\mathrm{F}(2,38)=78.73, p<0.001]$. The Bonferroni's multiple comparison showed a significant decrease in the post-intervention score compared with the pre-intervention score $(p<0.001)$, with the FU score also significantly lower than the pre-

\begin{tabular}{|c|c|c|c|c|}
\hline & \multicolumn{2}{|c|}{ Intervention group } & \multicolumn{2}{|c|}{ Control group } \\
\hline Factor & Mean & SD & Mean & \multicolumn{1}{|c|}{ SD } \\
\hline Age (years) & 65.15 & 3.65 & 67.21 & 5.18 \\
\hline CAPS score & 23.50 & 6.03 & 21.84 & 0.158 \\
\hline
\end{tabular}

ane-way analysis of variance.

HT: Horticultural Therapy; SE: Stress Education; SD: Standard Deviation; CAPS: Clinician-Administered Post-Traumatic Stress Disorder Scale

Table 1: Baseline demographic and clinical data of the participants.

\begin{tabular}{|c|c|c|c|c|c|c|c|c|c|}
\hline \multirow[b]{3}{*}{ Measures } & \multicolumn{4}{|c|}{ Intervention group } & \multicolumn{4}{|c|}{ Control group } & \multirow{4}{*}{$\begin{array}{c}p^{\mathrm{a}} \\
0.05\end{array}$} \\
\hline & \multicolumn{2}{|c|}{ Pre } & \multicolumn{2}{|c|}{ Post } & \multicolumn{2}{|c|}{ Pre } & \multicolumn{2}{|c|}{ Post } & \\
\hline & Mean & SD & Mean & SD & Mean & SD & Mean & SD & \\
\hline CAPS score & 23.50 & 6.03 & 6.60 & 5.25 & 21.84 & 4.83 & 10.63 & 8.90 & \\
\hline GDS score & 3.25 & 3.37 & 1.85 & 2.06 & 3.11 & 2.64 & 3.42 & 2.67 & 0.016 \\
\hline PTGI total score & 66.35 & 16.17 & 75.95 & 8.06 & 66.37 & 14.86 & 64.26 & 6.38 & 0.008 \\
\hline $\begin{array}{c}\text { WHO-QOL26 } \\
\text { Physical QOL score }\end{array}$ & 3.34 & 0.44 & 3.21 & 0.46 & 3.23 & 0.47 & 3.17 & 0.39 & 0.334 \\
\hline Psychological score & 3.11 & 0.30 & 3.46 & 0.53 & 3.04 & 0.49 & 2.99 & 0.62 & 0.010 \\
\hline Social score & 3.37 & 0.36 & 3.73 & 0.55 & 3.23 & 0.68 & 3.02 & 0.59 & 0.003 \\
\hline Environmental score & 2.92 & 0.48 & 3.31 & 0.48 & 2.80 & 0.56 & 2.72 & 0.68 & 0.021 \\
\hline Global score & 3.19 & 0.24 & 3.43 & 0.30 & 3.07 & 0.39 & 2.98 & 0.36 & 0.001 \\
\hline Salivary cortisol level & 2.29 & 2.45 & 1.53 & 1.63 & 2.49 & 1.94 & 3.49 & 2.28 & 0.025 \\
\hline
\end{tabular}

ane-way analyses of covariance with pre-post differences in psychological measures as dependent variables and pre-intervention scores as covariates (one-tailed). HT: Horticultural Therapy; SE: Stress Education; SD: Standard Deviation; CAPS: Clinician-Administered Post-Traumatic Stress Disorder Scale; GDS: Geriatric Depression Scale; PTGI: Posttraumatic Growth Inventory; WHO-QOL26: World Health Organization Quality Of Life 26

Table 2: Psychological measures pre- and post-intervention. 


\begin{tabular}{|c|c|c|c|c|c|c|}
\hline & \multicolumn{2}{|c|}{ Pre } & \multicolumn{2}{c|}{ Post } & \multicolumn{2}{c|}{ FU } \\
\hline Measures & Mean & SD & Mean & SD & Mean & SD \\
\hline CAPS score & 23.50 & 6.03 & 6.60 & 5.25 & 3.05 & 4.26 \\
\hline GDS score & 3.25 & 3.37 & 1.85 & 2.06 & 2.10 & 2.02 \\
\hline PTGI total score & 66.35 & 16.17 & 75.95 & 8.06 & 80.65 & 7.67 \\
\hline $\begin{array}{c}\text { WHO-QOL26 } \\
\text { Physical QOL score }\end{array}$ & 3.34 & 0.44 & 3.21 & 0.46 & 3.20 & 0.46 \\
\hline Psychological score & 3.11 & 0.30 & 3.46 & 0.53 & 3.59 & 0.41 \\
\hline Social score & 3.37 & 0.36 & 3.73 & 0.55 & 3.75 & 0.47 \\
\hline Environmental score & 2.92 & 0.48 & 3.31 & 0.48 & 3.36 & 0.48 \\
\hline Global score & 3.19 & 0.24 & 3.43 & 0.30 & 3.42 & 0.30 \\
\hline Salivary cortisol level & 2.29 & 2.45 & 1.53 & 1.63 & 1.66 & 1.62 \\
\hline
\end{tabular}

HT: Horticultural Therapy; SE: Stress Education; SD: Standard Deviation; CAPS: Clinician-Administered Post-Traumatic Stress Disorder Scale; GDS: Geriatric Depression Scale; PTGI: Posttraumatic Growth Inventory; WHO-QOL26: World Health Organization Quality Of Life 26

Table 3: Psychological changes of Intervention group (Pre, Post, and FU).

intervention score $(p<0.001)$ and post-intervention score $(p<0.01)$, confirming that this effect was sustained. GDS scores showed that the main effect of time (pre-intervention, post-intervention, and FU) was significant $[\mathrm{F}(2,38)=4.65, p<0.05]$. The Bonferroni's multiple comparison showed a significant lower in the post-intervention score compared with the pre-intervention score $(p<0.05)$, with the FU score also significantly lower than the pre-intervention score $(p<0.05)$, confirming that this effect was sustained. PTGI-J scores showed that the main effect of time (pre-intervention, post-intervention, and FU) was significant $[\mathrm{F}(2,38)=13.20, p<0.001]$. The Bonferroni's multiple comparison showed a significant higher in the post-intervention score compared with the pre-intervention score $(p<0.01)$, with the FU score also significantly higher than the pre-intervention score $(p<0.001)$ and post-intervention score $(p<0.01)$, confirming that this effect was sustained. WHO-QOL26 psychological QOL scores showed that the main effect of time (pre-intervention, post-intervention, and FU) was significant $[\mathrm{F}(2,38)=16.10, p<0.001]$. The Bonferroni's multiple comparison showed a significant higher in the post-intervention score compared with the pre-intervention score $(p<0.01)$, with the FU score also significantly higher than the pre-intervention score $(p<0.001)$ and post-intervention score $(p<0.05)$, confirming that this effect was sustained. WHO-QOL26 social QOL scores showed that the main effect of time (pre-intervention, post-intervention, and FU) was significant $[\mathrm{F}(2,38)=8.70, p<0.001]$. The Bonferroni's multiple comparison showed a significant higher in the post-intervention score compared with the pre-intervention score $(p<0.01)$, with the FU score also significantly higher than the pre-intervention score $(p<0.01)$. WHO-QOL26 environmental QOL scores showed that the main effect of time (pre-intervention, post-intervention, and $\mathrm{FU}$ ) was significant $[\mathrm{F}(2,38)=8.27, p<0.001]$. The Bonferroni's multiple comparison showed a significant higher in the post-intervention score compared with the pre-intervention score $(p<0.01)$, with the FU score also significantly higher than the pre-intervention score $(p<0.01)$. WHO-QOL26 global QOL scores showed that the main effect of time (pre-intervention, post-intervention, and $\mathrm{FU})$ was significant $[\mathrm{F}(2,38)=11.43, p<0.001]$. The Bonferroni's multiple comparison showed a significant higher in the post-intervention score compared with the pre-intervention score $(p<0.01)$, with the FU score also significantly higher than the preintervention score $(p<0.01)$.

Salivary cortisol level: Changes in salivary cortisol level in the intervention group are shown in Table 3 . The main effects was significant $[\mathrm{F}(2,38)=3.31 ; p<0.05]$. The Bonferroni's multiple comparison showed that the post-intervention cortisol level was significantly lower than the pre-intervention cortisol level $(p<0.05)$.

\section{Discussion}

The purpose of this study was to verify the reduction in the symptoms of earthquake-related stress in elderly women who live in disaster areas of the Pacific coast through HT intervention using psychological measures and salivary cortisol level, and to investigate the effect of HT on the symptoms of earthquake-related stress and the maintenance of its effect after a two-month follow-up period. The present study revealed that HT intervention affected the psychological changes and salivary cortisol level in elderly women with earthquakerelated stress and psychological effects remain effective over a period. These results are consistent with our hypothesis that HT may help elderly women with earthquake-related stress improve their mental and physical functioning affected due to the traumatic experience.

The intervention group showed improved CAPS scores, indicating that HT reduced PTSD symptoms. Also, this effect is sustained for a certain period. This finding was similar results to a previous study [21] and will extend the previous findings of the effect of HT on severe PTSD, by showing its efficacy with elderly women with earthquakerelated stress.

In the results of other psychological measures, the intervention group was improved GDS score, PTGI-J total scores, WHO-QOL26 score (psychological score, social score, environmental score, and global score), and salivary cortisol levels after HT intervention, indicating that HT reduced stress levels. This finding was related to salivary stress level are consistent with previous studies $[20,21,44]$. The intervention group showed improved GDS scores, indicating that HT decrease elderly depression. There are several HT studies for the elderly depression [45-47] and these studies reported that HT may reduce depression and stress. By our result, GDS score of intervention group decreased after HT intervention and GDS score almost sustained after FU. In other words, it is believed that HT is a method to improve a depression.

The intervention group showed improved PTGI-J scores, indicating that HT increased Posttraumatic Growth (PTG). The result of our previous horticultural intervention study reported PTGI total score in intervention group was improved and there is persistence of the effects for a certain period [21]. Previous study of PTG process suggests that people suffer emotional pain due to disruptions of their personal growth resulting from traumatic experiences ${ }^{51}$. However, people use PTG to cope in diverse ways, such as remembering their status before the event, referring to their own personality characteristics, relying on the support of others, and self-disclosing their own experiences with the negative event $[48,49]$. Previous study suggests that horticulture activity involves instinctive and creative action and leads to improvement of humanity [50]. Taking into consideration the factors mentioned above, it may be said that PTG and horticulture share a key feature. We think that participants of this intervention felt their own growth overlap with the growth process of the plant while cultivating the plants during two months. Additionally, this effect is sustained after FU periods. We also think that the result of PTG was reflected a psychological effect because horticulture work in our HT intervention was included fulfillment, pleasure, challenges, and a sense of accomplishment using plants such as flower and seedlings.

The intervention group showed improved WHO-QOL26 scores (psychological QOL score, social QOL score, environmental QOL score, and global QOL score) indicating that HT increased QOL. 
Previous studies suggest that HT improve QOL [51-53]. In our study, WHO-QOL26 physical QOL score did not have the change by the HT intervention. However, psychological QOL score, social QOL score, environmental QOL score, and global QOL score was significantly improve by HT intervention and these effects was sustained after FU periods. We think that the raising of the plant make challenging in everyday life and communication with the people around one. Additionally, we think the raising of the plant produces different changes life and the synergy that imposed by a plant improved the QOL.

The intervention group showed improved salivary cortisol levels, indicating that HT reduced stress. This finding was similar results to a previous study [20]. Cortisol is popular as indicator of psychological and physiological stress and salivary cortisol levels increase in people with PTSD symptoms. By this result, salivary cortisol levels in intervention group was significantly decreased after HT intervention, and salivary cortisol levels did not change after a follow-up compared to the postintervention. We think that the reduction of salivary cortisol level reflects that HT improve stress condition because the score of CAPS and GDS that used to measure stress-related psychological changes was improved after HT intervention and these effects is sustained after FU. For all of these reasons, the results of our present study suggest the possibility of HT as an effective intervention against the earthquakerelated stress.

The major limitation of this study was the small sample size. In the results of analysis of variance (ANOVA) for sensitivity, the effect size of this study was 0.45 and power was 0.8 , and a err prob was 0.05 . Therefore, a possible future direction would be to replicate and extend the results of current study with larger sample and a lighter (more casually controlled) trial design.

In conclusion, this study suggests that HT improve earthquakerelated stress such as depression of elderly women who live in disaster area of the Great East Japan earthquake and the psychological effects of HT was sustained. We believe HT may be able to suggest the possibility is one of the effective interventions for earthquake-related stress. We hope that it spread HT as a psychological support over the medium to long term in the natural disaster areas.

\section{Acknowledgements}

We thank study participants and all of our colleagues in Institute of Development, Ageing, and Cancer and in Tohoku University for their support. In addition, the authors thank Ms. Satomi Nishiyama, Ms. Ayaka Sato, Ms. Mio Sato, Ms. Kana Ohkiri, Ms. Ayumi Goto, Ms. Rei Takamatsu, Ms. Mayu Fujita, and Mr. Misaki Chiba for assisting with psychological testing. We also appreciate the contribution of Mrs. Taeko Shishido, a horticultural therapist who provided advice during the preparation of the intervention program. This study was supported by a Grant-in-Aid for Young Scientists (B) (KAKENHI 24730566) from the Ministry of Education, Culture, Sports, and Science to Dr. Y.K.

\section{References}

1. Canino G, Bravo M, Rubio-Stipec M (1990) The impact of disaster on mental health: prospective and retrospective analyses. Int J Ment Health 19: 51-69.

2. Madakasira S, O'Brien KF (1987) Acute posttraumatic stress disorder in victims of a natural disaster. J Nerv Ment Dis 175: 286-290.

3. Tyhurst JS (1957) Psychological and social aspects of civilian disaster. Can Med Assoc J 76: 385-393.

4. Yehuda R, Giller EL, Southwick SM, Lowy MT, Mason JW (1991) Hypothalamicpituitary-adrenal dysfunction in posttraumatic stress disorder. Biol Psychiatry 30: $1031-1048$

5. Fukuda S, Morimoto K, Mure K, Maruyama S (2000) Effect of the HanshinAwaji earthquake on posttraumatic stress, lifestyle changes, and cortisol levels of victims. Arch Environ Health 55: 121-125.
6. Galea S, Nandi A, Vlahov D (2005) The epidemiology of post-traumatic stress disorder after disasters. Epidemiol Rev 27: 78-91.

7. Kotozaki Y, Kawashima R (2012) Effects of the Higashi-Nihon earthquake: posttraumatic stress, psychological changes, and cortisol levels of survivors. PLoS One 7: e34612.

8. Detweiler MB, Sharma T, Lane S, Kim M, Johnson BC, et al. (2010) Practitioner forum: The case for using restorative natural environments in veterans rehabilitation programs. Fed Pract 1: 26-28.

9. Haller R, Kramer C (Eds.) (2006) Horticultural therapy methods: Making connections in health care, human service, and community programs. Binghamton, NY: The Haworth Press.

10. Mizuno-Matsumoto $Y$, Kobashi S, Hata Y, Ishikawa O, Asano F (2008) Horticultural therapy has beneficial effects on brain functions in cerebrovascular diseases. IC-MED Journal 2: 169-182.

11. Sugihara S, Aoyama $H$, Sugimoto M, Takeda S, Ikeda N, et al. (2006) The psychological cognitive and immunological effects of horticultural therapy on the elderly living in a nursing home. Japanese Journal of Geriatric Psychiatry 17: $967-975$.

12. Cimprich B (1993) Development of an intervention to restore attention to cancer patients. Cancer Nurs 12: 22-27.

13. Harzog T, Black A, Fountaine K, Knotts D (1997) Reflection and attentional recovery as distinct benefits of restorative environments. J Environ Psychol 17: $165-170$.

14. Porchey $P$ (2007) Horticultural therapy: How can it make a difference in your everyday life? Proc Fla State Hort Soc 120: 351-352.

15. Ulrich RS, Parson R (1992) Influences of passive experiences with plants on individual well-being and health. In: Relf D (Ed.), The role of horticulture in human well-being and social development. Portland: Timber Press. pp. 93-105.

16. Waliczek TM, Mattson RH, Zajicek JM (1996) Benefits of community gardening to quality of life issues. J Environ Hortic 14: 204-209.

17. Rodiek S (2002) Influence of an outdoor garden on mood and stress in older persons. J Ther Hortic 13: 13-21.

18. Langer EJ, Rodin J (1976) The effects of choice and enhanced personal responsibility for the aged: a field experiment in an institutional setting. J Pers Soc Psychol 34: 191-198.

19. Perrins-Margails N, Rugletic J, Schepis N, Stepanski H, Walsh M (2000) The immediate effects of group-based horticulture on the quality of life of persons with chronic mental illness. Occup Ther Ment Health 16: 15-30.

20. Van Den Berg AE, Custers MH (2011) Gardening promotes neuroendocrine and affective restoration from stress. J Health Psychol 16: 3-11.

21. Kotozaki Y (2013) The Psychological Effect of Horticultural Therapy Intervention on Earthquake-Related Stress in Women of Earthquake-Related Areas. J Transl Med Epidemiol 1: 1008.

22. Carr VJ, Lewin TJ, Webster RA, Hazell PL, Kenardy JA, et al. (1995) Psychosocial sequelae of the 1989 Newcastle earthquake: I. Community disaster experiences and psychological morbidity 6 months post-disaster. Psychol Med 25: 539-555.

23. Garrison CZ, Bryant ES, Addy CL, Spurrier PG, Freedy JR, et al. (1995) Posttraumatic stress disorder in adolescents after Hurricane Andrew. J Am Acad Child Adolesc Psychiatry 34: 1193-1201.

24. Armenian HK, Morikawa M, Melkonian AK, Hovanesian AP, Haroutunian N, et al. (2000) Loss as a determinant of PTSD in a cohort of adult survivors of the 1988 earthquake in Armenia: implications for policy. Acta Psychiatr Scand 102: $58-64$.

25. Ticehurst S, Webster RA, Carr VJ, Lewin TJ (1996) The psychosocial impact of an earthquake on the elderly. Int J Geriatr Psychiatry 11: 943-951.

26. Vernberg EM, Silverman WK, La Greca AM, Prinstein MJ (1996) Prediction of posttraumatic stress symptoms in children after hurricane Andrew. J Abnorm Psychol 105: 237-248.

27. Kwon YS, Maruyama S, Morimoto K (2001) Life events and posttraumatic stress in Hanshin-Awaji earthquake victims. Environ Health Prev Med 6: $97-$ 103.

28. Chan EY (2008) The untold stories of the Sichuan earthquake. Lancet 372 359-362. 
Citation: Kotozaki Y (2013) The Psychological Changes of Horticultural Therapy Intervention for Elderly Women of Earthquake-Related Areas. J Trauma Treat 3: 184. doi:10.4172/2167-1222.1000184

Page 6 of 6

29. Jia Z, Tian W, Liu W, Cao Y, Yan J, et al. (2010) Are the elderly more vulnerable to psychological impact of natural disaster? A population-based survey of adult survivors of the 2008 Sichuan earthquake. BMC Public Health 10: 172.

30. Liu A, Tan H, Zhou J, Li S, Yang T, et al. (2006) An epidemiologic study of posttraumatic stress disorder in flood victims in Hunan China. Can J Psychiatry 51: $350-354$

31. Sheehan DV, Lecrubier Y, Sheehan KH, Amorim P, Janavs J, et al. (1998) The Mini-International Neuropsychiatric Interview (M.I.N.I.): the development and validation of a structured diagnostic psychiatric interview for DSM-IV and ICD10. J Clin Psychiatry 59: 22-33.

32. Otsubo T, Tanaka K, Koda R, Shinoda J, Sano N, et al. (2005) Reliability and validity of Japanese version of the Mini-International Neuropsychiatric Interview. Psychiatry Clin Neurosci 59: 517-526.

33. Blake DD, Weathers FW, Nagy LM, Kaloupek DG, Gusman FD, et al. (1995) The development of a Clinician-Administered PTSD Scale. J Trauma Stress 8: $75-90$

34. Weathers FW, Keane TM, Davidson JR (2001) Clinician-administered PTSD scale: a review of the first ten years of research. Depress Anxiety 13: 132-156.

35. Asukai N, Tsuruta N, Saito A (2003) Psychometric properties of the Japaneselanguage version of the Clinician-Administered PTSD Scale for DSM-IV. Jpn J Trau Stress 1: 47-53.

36. Cwikel J, Ritchie K (1989) Screening for depression among the elderly in Israel: an assessment of the Short Geriatric Depression Scale (S-GDS). Isr J Med Sci 25: 131-137.

37. Niino N, Imaizumi T, Kawakami N. (1991) A Japanese translation of the geriatric depression scale short-form. Clin Gerontologist 10: 85-86.

38. Tedeschi RG, Calhoun LG (1996) The Posttraumatic Growth Inventory: measuring the positive legacy of trauma. J Trauma Stress 9: 455-471.

39. Taku K, Calhoun LG, Tedeschi RG, Gil-Rivas V, Kilmer RP, et al. (2007) Examining posttraumatic growth among Japanese university students. Anxiety Stress Coping 20: 353-367.

40. Tasaki M, Nakane M (1997) The WHO-QOL26. In: Health Division of Mental, Abuse Protection of Substance, editors. World Health Organization (in Japanese). Tokyo: Kaneko Shobou.
41. Fukuda S, Morimoto K (2001) Lifestyle, stress and cortisol response: Review I: Mental stress. Environ Health Prev Med 6: 9-14.

42. Riad-Fahmy D, Read GF, Walker RF (1983) Salivary steroid assays for assessing variation in endocrine activity. J Steroid Biochem 19: 265-272.

43. Toda M, Morimoto K, Nagasawa S, Kitamura K (2004) Effect of snack eating on sensitive salivary stress markers cortisol and chromogranin A. Environ Health Prev Med 9: 27-29.

44. O'Connor PJ, Corrigan DL (1987) Influence of short-term cycling on salivary cortisol levels. Med Sci Sports Exerc 19: 224-228.

45. Arashida E, Tsukagoshi S, Noda K, Kita T, Ohgama T, et al. (2007) Psychological and physiological verification of the therapeutic effects of horticultural activity mainly with herbs. J Hort Res. 6: 491-496.

46. Relf PD (1978) Horticulture as a recreational activity. J Am Health Care Assoc 4: 68-70.

47. Austin EN, Johnston YAM, Morgan L (2006) Community gardening in a Senior Center: Therapeutic Intervention to Improve the Health of Older Adults. Therapeutic Recreation Journal. 40: 48-56.

48. Rappe E, Kivelä SL (2005) Effects of garden visits on long-term care residents as related to depression. Hort Technology. 15: 298-303.

49. Tedeschi RG, Calhoun LG (2004) Posttraumatic growth: conceptual foundations and empirical evidence. Psychol Inq 15: 1-18.

50. Taku K, Cann A, Tedeschi RG, Calhoun LG (2009) Intrusive versus deliberate rumination in posttraumatic growth across US and Japanese samples. Anxiety Stress Coping 22: 129-136.

51. Matsuo, E (2008) Humanity in horticulture - healing and pleasure. Acta Hort (ISHS) 790: 39-44.

52. Barnicle T, Stoelzle Midden K (2003) The effects of a horticultural activity program on the psychological well-being of older people in a long-term care facility. HortTechnology. 13: 81-85.

53. Waliczek TM, Mattson RH, Zajicek JM (1996) Benefits of community gardening to quality of life issues. Journal of Environmental Horticulture. 14: 204-209. 